\title{
Caracterização de granjas suínas infectadas por Streptococcus suis*
}

\author{
Characterization of swine production farms infected by Streptococcus suis \\ Ana Elisa Del'Arco ${ }^{1}$, José Lúcio dos Santos ${ }^{2,3}$, José Eurico Faria ${ }^{3}$, Paula Dias Bevilacqua ${ }^{3}$, \\ Walter Vieira Guimarães ${ }^{2} \&$ Paulo Sérgio de Arruda Pinto ${ }^{3}$
}

\begin{abstract}
RESUMO
Neste trabalho foram estudados aspectos epidemiológicos das infecções causadas por Streptococcus suis, enfocando-se a caracterização de granjas acometidas por este agente no Brasil. Um questionário foi elaborado e enviado para propriedades infectadas que foram identificadas a partir do banco de dados do laboratório Microvet. Houve um retorno de 26,25\% dos questionários, onde se observou que em 73,8\% das propriedades, a suinocultura é a principal atividade desenvolvida, todas as granjas possuem sistema de criação intensivo convencional, $90,5 \%$ produzem no sistema de ciclo completo, $42,9 \%$ possuem entre 10 e 20 anos de existência e 38,1\% possuem entre 100 e 500 matrizes. Quanto à infecção, a idade mais observada de ocorrência foi o período de creche $(35,7 \%)$ e os sintomas nervosos foram os mais observados no momento do surto $(59,4 \%)$. O controle da infecção se fez na maior parte das granjas por penicilina $(26,2 \%)$ e o uso de vacinas ainda não é prática em todas as granjas. Apesar do reduzido número de questionários recebidos, os dados mostram uma tendência de que as granjas brasileiras, infectadas pelo $S$. suis, trabalham com o sistema intensivo de produção, sendo a maioria granjas de tamanho médio a grande e com mais de 10 anos de existência. Além disso, há uma tendência de que o surto da doença ocorra, em sua maioria, na fase de creche e o seu controle é feito por antibioticoterapia à base principalmente de penicilina.
\end{abstract}

Descritores: suínos, Streptococcus suis, questionário.

\section{ABSTRACT}

In this work, it were studied epidemic aspects of the infections caused by Streptococcus suis, focusing the characterization of farms infected in Brazil. A questionnaire was elaborated and mailed for properties which one were identified from the database of the laboratory Microvet. There was a return of $26.25 \%$ of the questionnaires where it was observed that in $73.8 \%$ of the properties, the swine production is the main developed activity, was observed that all the farms are producing pigs in conventional intensive system, $90.5 \%$ produce in the system of it completes cycle, $42.9 \%$ have between 10 and 20 years of existence and $38.1 \%$ have between 100 and 500 sows. With relationship to the infection, the observed age of occurrence was the nursing period $(35.7 \%)$ and the nervous symptoms were the more observed in the moment of the infection (59.4\%). The control of infection was made in most of the farms with penicillin (26.2\%) and the use of vaccines is not common in all the farms yet. In spite of the reduced number of received questionnaires, the data show a tendency that Brazilian farms, infected by S. suis, work with the intensive system of production, most farms have medium to big size and have more than 10 years of existence. Besides, there is a tendency that the outbreak of the disease happens, in its majority, in the nursery and its control is made mainly by antibiotics of penicillin base.

Key words: pigs, Streptococcus suis, questionnaire. 


\section{INTRODUÇÃO}

O Streptococcus suis é uma bactéria causadora de diversas doenças em suínos, como pneumonia, artrite, septicemia e meningite. Já foram identificados 35 sorotipos, sendo o sorotipo 2 considerado o mais virulento $[27,33]$. Por fazer parte da flora normal de suínos, e por não poder ser erradicado de um rebanho [4,14]; pesquisadores do mundo todo têm direcionado seus esforços para entender quais são os fatores importantes na sua patogenia, para que mecanismos de controle mais eficientes sejam desenvolvidos $[8,9,18,34]$. Durante a última década, a indústria suinícola direcionou seus maiores esforços para a produção de rebanhos com baixo nível de doença. Mas, nos últimos anos, o Streptococcus suis tem emergido como um importante patógeno na suinocultura mundial, particularmente em rebanhos de elevado estado de sanidade. Segundo o relatório final do Encontro Técnico da ABRAVES nacional sobre meningite estreptocócica em suínos, uma das prioridades para o Brasil seria a caracterização dos sorotipos do Streptococcus suis, bem como o estudo dos fatores de risco relacionados com a infecção [36]. No Brasil, os primeiros trabalhos para caracterização deste agente patogênico e da sua infecção começaram a ser desenvolvidos, principalmente, após o ano de 1997. Hoje, já se conhece a distribuição de seus principais sorotipos e algumas características da sua infecção $[10,28,29]$.

O objetivo do trabalho foi a caracterização de granjas acometidas pelo $S$. suis no Brasil.

\section{MATERIAIS E MÉTODOS}

A partir do banco de dados do laboratório Microvet (Microbiologia Veterinária Especial Ltda, Viçosa, MG), foram identificadas as propriedades, de várias regiões do país, que enviaram animais ou órgãos para identificação do agente patogênico e tiveram isolamento de $S$. suis, durante os anos de 1995 a 2001. A partir de então se elaborou um questionário para ser enviado aos proprietários das granjas positivas para este agente, cuja finalidade era de pesquisar dados epidemiológicos referentes ao estudo da infecção por S. suis. Enviou-se o questionário, via correio, para 160 propriedades. Não houve possibilidade de envio para todas as propriedades (191) por falta de endereço de algumas delas. O envelope com o questionário continha, ainda, correspondência explicativa da importância e finalidade do questionário e envelope menor selado e sobrescrito para devolução via correio. Por telefone, manteve-se contato com os proprietários, solicitando o preenchimento e a devolução dos questionários.

\section{RESULTADOS}

A caracterização dos rebanhos suínos foi estudada a partir dos resultados dos questionários recebidos. Houve um retorno de 42 dos 160 questionários $(26,25 \%)$ durante janeiro a agosto de 2001. Estes questionários representavam 7 estados brasileiros, sendo que houve maior retorno de Minas Gerais $(54,7 \%)$, seguido de Paraná (19,0\%), São Paulo (14,3\%), Rio Grande do Sul (4,8\%), Mato Grosso do Sul $(2,4 \%)$, Santa Catarina $(2,4 \%)$ e Pernambuco $(2,4 \%)$.

\section{Caracterização das granjas e dos rebanhos}

Pôde-se observar que, em 73,8\% das propriedades, a suinocultura era a principal atividade e o sistema de criação era intensivo convencional para todas as granjas. Em 90,5\% das granjas, a produção era de ciclo completo, $7,1 \%$ era produção de leitões e $2,4 \%$ (1 granja) era terminação. Segundo o tempo de produção de suínos nas granjas, $42,9 \%$ possuem entre 10 a 20 anos de produção, seguidas de granjas com mais de 20 anos (26,2\%), 5 anos $(19,0 \%)$ e de granjas entre 5 e 10 anos $(11,9 \%)$.

Em relação ao tamanho da granja, observouse que $38,1 \%$ possuem entre 100 e 500 matrizes, seguidos de granjas entre 500 e $1.000(23,8 \%)$, mais de $1.000(19,0 \%)$ e entre 30 e 100 matrizes $(16,7 \%)$.

Observou-se que, das 42 propriedades, $69,0 \%$ estão distantes de outra granja em mais de $1.000 \mathrm{~m}$, seguidas de granjas distantes em $500 \mathrm{~m}$ e entre $500 \mathrm{e}$ $1.000 \mathrm{~m}(16,7 ; 11,9 \%$, respectivamente).

No momento da desmama dos leitões, os lotes são formados com base na idade dos animais desmamados $(83,3 \%)$, ou mesmo utilizando outros parâmetros associados à idade, como tamanho $(14,3 \%)$ ou leitegada $(2,4 \%)$.

Das 42 granjas, somente 2 (4,8\%) são livres de doenças respiratórias. Vinte e nove granjas possuem pneumonia enzoótica na granja $(69,0 \%)$ e em 24 destas 29 granjas ocorrem outras doenças respiratórias concomitantemente. Em 11 granjas (26,2\%) ocorrem doenças respiratórias, mas são livres de pneumonia enzoótica. 


\section{Caracterização da infecção por Streptococcus suis}

Os meses mais relacionados com a ocorrência de surtos por $S$. suis foram maio, junho e julho $(35,7 \%$, $35,7 \%$ e 28,6\% respectivamente). Em 14 dos 42 questionários $(33,3 \%)$ os proprietários observaram a ocorrência de surtos de $S$. suis em qualquer mês do ano.

Quanto à idade dos animais mais freqüentemente acometidos por $S$. suis, em $35,7 \%$ das granjas houve maior acometimento de animais da creche e recria, seguido de animais da creche somente $(21,4 \%)$; creche, recria e terminação $(14,3 \%)$; recria $(11,9 \%)$; período de maternidade associado com creche ou recria ou ambas (7,1\%); períodos de recria e terminação $(4,8 \%)$ e períodos de creche e terminação $(2,4 \%)$. Um questionário não respondeu esta questão $(2,4 \%)$.

Os sintomas mais reconhecidos, na época do surto da doença na granja, foram os nervosos $(59,4 \%)$, seguidos de sintomas articulares $(4,8 \%)$ e respiratórios $(4,8 \%)$. Houve relatos de vários desses sintomas ocorrendo conjuntamente $(26,2 \%)$.

Dentre a sintomatologia nervosa, os sinais clínicos mais observados foram: incoordenação (em $95,2 \%$ dos casos), pedalagem e opstotomus $(85,7 \%)$ e tremor muscular $(57,1 \%)$. Na ocasião dos surtos, não houve acometimento de outras espécies animais nas propriedades.

Quanto ao tratamento, as drogas mais utilizadas foram penicilina $(26,2 \%)$, amoxicilina $(21,4 \%)$ ou ambas $(21,4 \%)$. Em 42,9\% das granjas a medicação controlou a infecção causada por $S$. suis, em $28,6 \%$ houve controle durante o seu uso das drogas, em 14,3\% houve a transferência da infecção para os mais velhos e em $7,1 \%$ não houve controle com antibioticoterapia.

Em 23 das 42 granjas $(57,1 \%)$ ocorre o uso de vacinas para controle de $S$. suis, e dentre estas, em 13 $(56,6 \%)$ houve o controle da doença somente com seu uso. Em $9(39,1 \%)$ houve controle com o uso de vacinas, juntamente com o uso de antibióticos e em apenas uma a vacina não controlou a doença $(4,3 \%)$.

\section{DISCUSSÃO}

A utilização da metodologia de questionário via correio tem sido mostrada como uma ferramenta importante para investigações epidemiológicas $[5,16,21,22,25]$ mas sua utilização na Medicina Veterinária ainda é pequena. A taxa média de resposta de questionários enviados por correio fica em torno de $59 \%$ \pm 20 e que fatores como, envio de envelope de resposta já selado e manutenção de contato por telefone, podem aumentar esta taxa de resposta [5]. O observado neste trabalho é que apesar de terem sido realizados todos os passos para que houvesse uma taxa de resposta satisfatória, esta ficou abaixo da média esperada de 59\%. Além disso, no Brasil, não existe o costume de realizações de inquéritos epidemiológicos através de questionários enviados por correio, nem na área da Medicina Humana, nem na Veterinária. Desta forma, a falta de costume pode levar a uma não conscientização da importância da realização deste tipo de trabalho (R. C. Dias, comunicação pessoal). Além disso, por produtores rurais terem sido o público alvo deste inquérito, talvez a aplicação do questionário pessoalmente ou por telefone poderia ter trazido maior participação. Mas esta metodologia inviabilizaria o presente trabalho, já que foram aplicados questionários nas mais variadas regiões do país, o que aumentaria demasiadamente os custos desta aplicação. Apesar disso, os dados aqui apresentados podem mostrar uma tendência da produção brasileira e auxiliar em possíveis investigações futuras.

Pôde-se observar que a maioria das propriedades que responderam ao questionário se concentra no Estado de Minas Gerais, sendo que a maioria das propriedades identificadas no momento de envio deste questionário se localizava neste estado também. Esta grande ocorrência pode ser explicada pelo fato de o Laboratório Microvet estar localizado neste Estado, condição que pode tornar seus serviços de isolamento e diagnóstico mais conhecidos nesta região, além do custo de envio de material para diagnóstico oriundo de regiões muito distantes ser maior para os produtores.

Outro fato importante, para o esclarecimento deste maior número, é o crescimento, também em Minas Gerais, de uma suinocultura desenvolvida que tem, cada vez mais, empregado alta tecnologia em seu processo de produção. $\mathrm{O}$ emprego desta alta tecnologia está geralmente associado a uma maior preocupação com o processo de produção e maior vigilância sanitária dos rebanhos, com o intuito de combater, cada vez mais cedo e com maior eficácia, surtos de doenças na população suína.

Diversos estudos têm mostrado a importância do controle de doenças para uma produção eficiente e para manutenção do produtor no mercado, uma vez que os processos infecciosos causam grandes pre- 
juízos, diminuem a margem de lucro e levam a uma menor competitividade pelo mercado consumidor $[11,12,15,30,39,40]$. Este fato pode ser fundamentado observando que todas as propriedades que responderam, trabalham com o sistema de produção intensivo convencional e ser a suinocultura a principal atividade desenvolvida na propriedade.

Quanto ao tempo de produção, observa-se que a grande maioria das granjas possui mais de 10 anos de produção. Isto caracteriza granjas mais antigas, em que a possibilidade de contaminação por agentes infecciosos é maior que em granjas mais novas. Nos sistemas de produção de ciclo completo, produção de leitões e terminadores, ocorrem entradas de animais com uma certa freqüência, o que possibilita a veiculação de novos agentes infecciosos. Assim, em granjas mais antigas, por já terem ocorrido maior número de entradas de animais e outros veículos de contaminação, a probabilidade é destas granjas possuírem mais problemas infecciosos do que granjas mais novas [3].

Para a disseminação de doenças numa população, os fatores de maior importância para esta ocorrência são a movimentação de animais e a distância de granjas vizinhas. Quanto à movimentação de animais, a entrada destes numa região pode ser de grande risco, caso sejam portadores de algum patógeno e quanto maior esta movimentação em uma região maior a possibilidade de circulação de um agente patogênico.

Quando se trata de densidade de animais, a distância entre granjas vizinhas é um dado de grande importância, pois, além de estar relacionada a este fator, também existem os riscos de contaminação de uma granja para outra por aerossóis, movimentação de pessoas e mesmo de veículos [23].

A transmissão do Streptococcus suis tipo 2 por aerossóis entre suínos já foi demonstrada [6], reforçando a importância deste fator no processo de disseminação do agente dentro de um rebanho e também entre rebanhos vizinhos, a depender da distância entre eles. Considerando uma distância de $3 \mathrm{~km}$, o risco de disseminação de doenças bacterianas é muito baixo [23], mas este risco, e, conseqüentemente, a distância entre granjas, pode diminuir quando se controla a entrada não só de animais, como também de pessoas e veículos, além da presença de barreiras físicas entre elas.

Como a produção de suínos tecnificada brasileira se caracteriza por granjas com o controle da en- trada de animais e de pessoas, o risco de infecção entre rebanhos vizinhos torna-se menor.

Quando se observa o manejo dos animais dentro da suinocultura intensiva, a fase de creche é um período crítico, pela ocorrência de problemas de desempenho e de ordem sanitária. Os leitões ao desmame são submetidos a vários fatores estressantes de ordem nutricional (mudança da dieta), social (formação de novas hierarquias), ambiental e de manejo (transferência dos leitões para ambiente desconhecido) que favorecem a proliferação de agentes infecciosos [24].

Além disso, sabe-se que fatores estressantes podem agravar ou mesmo desencadear um processo clínico devido à presença de um microrganismo patogênico no rebanho. $\mathrm{O}$ stress faz com que haja maior liberação de cortisol, que por sua vez interfere na capacidade funcional do sistema imune do animal [37]. A formação de lotes de leitões neste período é de extrema importância, pois, a mistura de animais de idade e tamanhos diferentes nesta etapa pode ter sérias consequiências, como efeitos mais acentuados do estresse causado por brigas por definição de hierarquia e transmissão de patógenos para animais com sistema imunológico menos desafiado.

Em se tratando de desafio ao sistema imunológico, o fato de uma granja possuir outras doenças concomitantes pode levar ao aparecimento mais freqüente de infecções secundárias. Um estudo [32] demonstrou que tanto a rinite atrófica quanto a presença de pneumonia estão amplamente disseminadas nos rebanhos de suínos nos estados do sul do país (98,4\% dos rebanhos para rinite e $100 \%$ para pneumonia). Estes dados mostram a importância destas doenças pelo alto grau de disseminação que possuem em alguns rebanhos brasileiros.

A pneumonia enzoótica é uma das doenças mais comuns no mundo e de maior importância econômica. Além de ter um efeito imunodepressor, o Mycoplasma hyopneumoniae acarreta a perda da capacidade de clearance do trato respiratório superior, o que predispõe a infecções bacterianas secundárias [13]. Desta forma, a presença deste agente num rebanho, poderia predispor o aparecimento de surtos causados por outros agentes infecciosos, inclusive o S. suis.

Com relação aos dados de distribuição desta doença nos diversos meses do ano, estes devem ser 
analisados com cautela. Poderia-se esperar que a demanda laboratorial estivesse diretamente ligada ao aparecimento de casos clínicos no campo e com isto haveria uma maior necessidade de diagnóstico. Desta forma espera-se que uma freqüência maior de casos de doença no campo esteja diretamente relacionada com o aumento observado no número de diagnósticos realizados por um laboratório da área. Mas existem outros fatores que podem influenciar, como o fato de o laboratório associado à pesquisa ser ou não conhecido em outras regiões do país, e também a participação de assistência técnica local, que pode tratar de casos de doença com antibioticoterapia, sem necessariamente envio de material para diagnóstico preciso.

A baixa frequiência nos meses de janeiro, fevereiro e março podem ser devido à maior quantidade de vendas de animais durante o mês de dezembro, por causa dos feriados de fim-de-ano. Isto faz com que nestes meses seqüentes a densidade de animais na granja seja menor e, conseqüentemente, haja menos casos de doença. [26] isolaram o $S$. suis em todos os meses do ano e um discreto aumento dos isolado ocorreu nos períodos de outono e inverno.

Os dados apresentados, em relação à idade mais acometida pela infecção, demonstram uma maior ocorrência da infecção por $S$. suis na creche, seguido da recria, o que está de acordo com o mesmo período de infecção relatado na literatura, sendo de 4 a 12 semanas de idade, com pico de ocorrência em torno de 42 dias [27,33].

Houve relatos de ocorrência de infecção na terminação, o que não é um dado muito comum. Uma provável causa do aparecimento tardio destas infecções é a utilização da prática de medicação estratégica em suinocultura para controle de agentes infecciosos presentes na granja. Esta prática consiste em administrar antimicrobianos na ração pelo período de uma semana antes da idade de maior ocorrência do surto de determinada doença. Esta medicação pode estar controlando o $S$. suis na faixa etária em que ele é relatado como sendo mais freqüentemente encontrado, ou seja, em torno de 42 dias de idade. Mas com o tempo, as bactérias que não foram eliminadas por esta medicação se multiplicam novamente e causam o aparecimento de casos clínicos, neste caso em idades superiores ao controle realizado com a medicação estratégica.

Quanto aos sintomas apresentados na ocasião do surto, a sintomatologia nervosa foi a mais freqüente, o que está de acordo com os dados da literatura em que esta sintomatologia é a mais característica de processos infecciosos causados pelo S. suis $[17,27,33]$.

Quanto ao tratamento, observa-se que a droga mais utilizada continua sendo a penicilina, preconizada na literatura como mais eficaz no combate à infecção [27]. Mas casos de resistência à penicilina têm sido relatados desde 1980, o que impõe cada vez mais a necessidade de fazer antibiogramas para conhecer a sensibilidade de diferentes amostras isoladas [35]. Outros relatos de aumento de resistência a diversos antibióticos são encontrados na literatura $[1,2,38]$.

O uso de vacinas para controle do $S$. suis ainda não alcançou os resultados almejados. Apesar de vários estudos realizados com o intuito de esclarecer quais mecanismos, relacionados à resposta imunológica (celular e humoral), são importantes para a proteção do animal, ainda não se conhecem com clareza os caminhos necessários para a produção de vacinas realmente eficazes [7,19,20,31].

\section{CONCLUSÕES}

Apesar do reduzido número de questionários recebidos, os dados mostram uma tendência de que as granjas brasileiras, infectadas pelo $S$. suis, trabalham com o sistema intensivo de produção, sendo a maioria granjas de tamanho médio a grande e com mais de 10 anos de existência. Além disso, há uma tendência de que o surto da doença ocorra, em sua maioria, na fase de creche e o seu controle é feito por antibioticoterapia à base principalmente de penicilina.

Agradecimentos. Os autores agradecem o apoio do PADCT/MCT, Brasil.

Comunicação pessoal: Roberta Costa Dias [tepoz@uol.com.br]. 


\section{REFERÊNCIAS}

1 Aarestrup F. M., Jorsal S. E. \& Jensen N. E. 1998. Serological characterization and antimicrobial susceptibility of Streptococcus suis isolates form diagnostic samples in Denmark during 1995 and 1996. Veterinary Microbiology. 60: 5966.

2 Aarestrup F. M., Rasmussen S. R. \& Artusson K. \& Jensen N. E. 1998. Trends in the resistance to antimicrobial agents of Streptococcus suis isolates form Denmark and Sweden. Veterinary Microbiology. 63: 71-80.

3 Alexander T. J. L. \& Harris D. L. 1992. Methods of Disease Control. In: Leman A. D., Straw B. E., Mengeling W. L., D’Allaire S. \& Taylor D. J. (Eds). Diseases of Swine. 7th edn. Ames: Iowa State University Press, pp. 808-836.

4 Amass S. F., Sanmiguel P. \& Clark L.K. 1997. Demonstration of vertical transmission of Streptococcus suis in Swine by genomic fingerprinting. Journal of Clinical Microbiology. 35: 1595-1596.

5 Asch D. A., Jedrziewski M. K. \& Christakis N. A. 1997. Responses rates to mail surveys published in Medical Journals Journal of Clinical Epidemiology. 50: 1129-1136.

6 Berthelot-Hérault F., Gottschalk M., Labbé A., Cariolet R. \& Kobish M. 2001. Experimental airbone transmission of Streptococcus suis capsular type 2 in pigs. Veterinary Microbiology. 82: 69-80.

7 Busque P., Higgins R., Caya F. \& Quessy S. 1997. Immunization of pigs against Streptococcus suis serotype 2 infection using a live avirulent strain. Canadian Journal of Veterinary Research. 61: 275-279.

8 Charland N., Kellens J. T. C., Caya F. \& Gottschalk M. 1995. Aglutination of Streptococcus suis by sialic acid-biding lectins. Journal Clinical Microbiology. 33: 2220-2221.

9 Charland N., Harel J., Kobish M., Lacasse S. \& Gottschalk M. 1998. Streptococcus suis serotype 2 mutants deficient in capsular expression. Microbiology. 144: 325-332.

10 Costa A. T. R., Reis R., Lobato F. C. \& Viegas V. 2001. Sorotipificação e avaliação da patogenicidade de amostras de Streptococcus suis isoladas de suínos. In: X Congresso Brasileiro de Veterinários Especialistas em Suínos (Porto Alegre, Brasil). Cd-room.

11 Cuevas L. 1998. The epidemiology of pig diseases In: 15th International Pig Veterinary Society Congress.v.1. (Birmingham, Inglaterra). pp. 87-93.

12 Dalla Costa O. A., Mores N., Sobestiansky J., Barioni W. J., Piffer I. A., Pedroso De Paiva D., Guzzo R., Lima G. J. M. M. \& Perdomo C. C. 1999. Estudo ecopatológico nas fases de crescimento e terminação: fatores de risco associados à rinite atrófica progressiva e a pneumonias. In: IX Congresso Brasileiro de Veterinários Especialistas em Suínos (Belo Horizonte, Brasil). pp.169-170.

13 Done H. S. 1996. Enzootic pneumonia (Mycoplasmosis) revisited. The Pig Journal. 38: 40-61.

14 Dritz S. S., Chengappa M. M., Nelssen J. L., Tokach M. D., Goodband R. D., Nietfeld J. C. \& Staats J. J. 1996. Growth and microbial flora of nonmedicated segregated, early weaned pigs, from a commercial swine operation. Journal of American Veterinary Medical Association.. 208: 711-715.

15 Dritz S. S., Owen, K. Q., Goodband R. D., Nelssen J. L., Tokach M. D., Chengappa M. M. \& Blecha F. 1996. Influence of lipopolysaccharide-induced immune challenge and diet complexity on growth performance and acute-phase protein production in segregated early-weaned pigs. Journal Animal Science. 74: 1620-1628.

16 Etter J. F. \& Perneger T. V. 1997. Analysis of non-response bias in a mailed health survey. Journal of Clinical Epidemiology. 50: 1123-1128.

17 Gogolewski R. P., Cook R. W. \& O’Connell C. J. 1990. Streptococcus suis serotypes associated with disease in weaned pigs. Australian Veterinary Journal. 67: 202-204.

18 Gottschalk M. \& Segura M. 2000. The patogenesis of meningitis caused by Streptococcus suis: the unresolved questions. Veterinary Microbiology. 76: 259-272.

19 Holt M. E., Emright M. R. \& Alexander T. J. L. 1989. Studies of the protective effect of different fractions of sera form pigs immune to Streptococcus suis type 2 infection. Journal of Comparative Pathology. 100: 435-442.

20 Jacobs A. A. C., Van Den Berg A. J. G. \& Loeffen P. L. W. 1996. Protection of experimentally infected pigs by suilisyn, the thiol-activated haemolysin of Streptococcus suis. Veterinary Record. 7: 225-228.

21 Kotaniemi J. T., Hassi J., Jonsson, E. Laitinen L. A., Sovijarvi A. R. \& Lundback B. 1999. Does non-responder bias have a significant effect on the results in a postal questionnaire study? American Journal of Epidemiology. 149: $1057-62$. 
22 Larroque B., Kaminski M., Bouvier-Colle M.H. \& Hollebecque V. 1999. Participation in a mail survey: role of repeated mailings and characteristics of nonrespondents among recent mothers. Pediatric and Perinatal Epidemiology. 13:218-233.

23 Madec F. 2001. Biosecurity of pig units: a major issue for herd health maintenance. In: X Congresso Brasileiro de Veterinários Especialistas em Suínos (Porto Alegre, Brasil). Cd-Rom.

24 Mores N. \& Amaral A. L. 2001. Patologias associadas ao desmame. In: X Congresso Brasileiro de Veterinários Especialistas em Suínos (Porto Alegre, Brasil). Cd-Rom.

25 Pearce G. P. 1999. Epidemiology of enteric disease in grower-finisher pigs: a postal survey of pig producers in England. Veterinary Record. 144: 338-342.

26 Reams R. Y., Harrington D. D., Glickman L. T., Thacker H. L. \& Terry L. B. 1996. Multiple serotypes and strains of Streptococcus suis in naturally infected swine herds. Journal of Veterinary Diagnostic Investigation. 8: 119-121.

27 Sanford S. E. \& Higgins R. 1992. Streptococcal diseases. In: Leman A. D., Straw B. E., Mengeling W. L., D’Allaire S.\& Taylor D. J. (Eds). Diseases of Swine. 7th edn. Ames: Iowa State University Press, pp.588-590.

28 Santos J. L., Del'Arco A. E., Ribeiro M. C. E. \& Guimarães W. V. 1999. Distribuição de sorotipos de Streptococcus suis em suínos clinicamente doentes no Brasil. In: IX Congresso Brasileiro de Veterinários Especialistas em Suínos (Belo Horizonte, Brasil). pp.239-240.

29 Santos J. L., Del'Arco A. E., Ribeiro M. C. E. \& Guimarães W. 2000. Occurrence of Streptococcus suis serotypes in pigs in Brazil. In: The 16th International Pig Veterinary Society Congress (Melbourne, Austrália). p.536.

30 Sauber T. E., Stahly T. S. \& Nonnecke B. J. 1999. Effect of level of chronic immune system activation on the lactational performance of sows. Journal of Animal Science. 77: 1985-1993.

31 Sepulveda E. M. C., Altman E., Kobisch M., Allaire S. D. \& Gottschalk M. 1996. Detection of antibodies against Streptococcus suis capsular type 2 using a purified polysaccharide antigen-based indirect ELISA. Veterinary Microbiology. 52: 113-125.

32 Sobestiansky J., Dalla Costa O. A., Mores N., Barioni W.J., Piffer I. A. \& Pedroso-De-Paiva D. 1999. Estudos ecopatológicos das doenças respiratórias: prevalência de rinite atrófica e de pneumonia nas fases de crescimento e terminação na região Sul do Brasil. In: IX Congresso Brasileiro de Veterinários Especialistas em Suínos (Belo Horizonte, Brasil). pp.171-172.

33 Staats J. J., Feder I., Okwumabua O. \& Chengappa M. M. 1997. Streptococcus suis: past and present. Veterinary Research Communications. 21:381-407.

34 Staats J. J., Plattner B. L., Nietfeld J., Dritz S. \& Chengappa M. M. 1998. Use of ribotyping and hemolysin activity to identify highly virulent Streptococcus suis type 2 isolates. Journal of Clinical Microbiology. 36: 15-19.

35 Turgeon P. I., Higgins R., Gottshalk, M. \& Beaudoin M. 1994. Antimicrobial susceptibility of Streptococcus suis isolates. British Veterinary Journal.150: 263-269

36 Vaz A. K. 1999. Relatório da reunião final. In: Simpósio Sobre Meningite Estreptocóccica Suína e Pleuropneumonia Suína (Lages, Brasil). pp.42-44.

37 Wallgren P., Wilén I. \& Fossum C. 1994. Influence of experimental induced endogenous production of cortisol on the immune capacity in swine. Veterinary Immunology and Immunopathology. 42: 301-316.

38 Wasteson Y., Hoie S. \& Oberts M. C. 1994. Characterization of antibiotic resistance in Streptococcus suis. Veterinary Microbiology. 41: 41-49.

39 Williams N. H., Stahly T. S. \& Zimmerman D. R. 1997. Effect of chronic immune system activation on body nitrogen retention, partial efficiency of lysine utilization, and lysine needs of pigs. Journal Animal Science. 75: 2472-2480.

40 Williams N. H., Stahly T. S. \& Zimmerman D. R. 1997. Effect of level of chronic immune system activation on the growth and dietary lysine needs of pigs fed from 6 to $112 \mathrm{~kg}$. Journal of Animal Science. 75: 2481-2496. 